\title{
Dissecting altered functional engagement in TBI and other patient groups through connectivity analysis: one goal, many paths (a response to Hillary)
}

\author{
Gary R. Turner ${ }^{1 *}$, Anthony R. Mclntosh ${ }^{2,3}$ and Brian Levine ${ }^{2,3,4}$ \\ 1 Faculty of Health, Department of Psychology, York University, Toronto, ON, Canada \\ 2 Department of Psychology, University of Toronto, Toronto, ON, Canada \\ ${ }^{3}$ Rotman Research Institute, Baycrest Center for Geriatric Care, Toronto, ON, Canada \\ 4 Faculty of Medicine, Department of Neurology, University of Toronto, Toronto, ON, Canada \\ ${ }^{*}$ Correspondence: grturner@yorku.ca
}

\section{A commentary on}

Determining the nature of prefrontal cortex recruitment after traumatic brain injury: a response to Turner

by Hillary, F. G. Front. Sys. Neurosci. 5:24. doi: 10.3389/fnsys.2011.00024

We are grateful to Hillary for his detailed commentary on our article (Turner et al., 2011), which gives an opportunity to clarify some of the main points of the article. We are pleased that Hillary underscores the main interpretation of our data, which is that augmented neural activity is compensatory and does not reflect brain reorganization, and that the behaviorally relevant regions recruited in TBI are also functionally relevant in healthy adults at a higher level of task difficulty. Hillary (2011) notes that the innovation of this altered functional engagement hypothesis is "not entirely clear." We do not claim ownership of the intellectual history of this notion, which has been invoked in many contexts, including in theories of natural recovery, and rehabilitation in brain injury (Backman and Dixon, 1992; Robertson and Murre, 1999). Indeed, empirical support for this notion was demonstrated in the early days of functional neuroimaging (Wood et al., 1980) in patients with stroke (Weiller et al., 1992; Engelien et al., 1995; Buckner et al., 1996) and dementia (Bookheimer et al., 2000). We showed similar findings in a case study of TBI more than a decade ago (Levine et al., 1998). The altered functional engagement idea has also been extensively discussed in the neurocognitive aging literature (e.g., Cabeza et al., 1997; Reuter-Lorenz et al., 2000; Rypma and D'Esposito, 2000). As we noted in our paper, this interpretation does not necessarily follow from activation maps; it is supported by analysis of corre- lations between brain signal and behavior, between brain signals from different regions (i.e., functional connectivity), and between functional connectivity and behavior. These concepts were empirically tested in patients with Alzheimer's disease as early as 2003 with the seed-behavioral PLS technique (Grady et al., 2003a,b), which served as a model for our paper.

Hillary made several criticisms of our paper, which we will address in order:

(1) “The authors define 'activation' in their study as the relationship between the fMRI signal and task accuracy; this the fMRI signal is modeled solely as it relates to task accuracy."

It is inappropriate to define the correlation of task accuracy to fMRI signal as "activation" and in fact we did not do so in our paper. Moreover, the assertion that we modeled the fMRI signal solely in relation to task accuracy is incorrect. In behavioral PLS and seed-behavioral PLS, the mean BOLD response to the events of interest (task demands, seed-connectivity) are extracted for each subject, then correlated with accuracy across subjects.

(2) "The authors are also unlikely to observe all possible neural resources that are differentially recruited after injury. For example, there may be neural responses that hold non-linear relationships with accuracy or are related only to reaction time."

We admit to being unable to observe "all possible neural resources that are differentially recruited after injury," and we consider any such claim to be ludicrous. Our paper was focused on the change in functional connectivity as it relates to group and behavior. Thus our seed-behavior PLS delineated patterns of connectivity that differentially related to behavior across the two groups. Turning to reaction time, this was not a relevant measure for the working memory task that we used, which is a well-validated and established task of differential executive demands in working memory in the fMRI environment. These differential demands are determined at the encoding (number of items) and manipulation (alphabetize versus retain) phases of the task. Reaction time at the probe phase is irrelevant to the executive demand manipulations in this task and therefore would not have been an appropriate measure to include in our analyses.

(3) "The problem is that the authors have no way to identify prefrontal involvement after TBI that is not correlated with performance, an outcome that could be a candidate for brain reorganization."

This statement is incorrect. First, we conducted a whole-brain analysis of taskrelated activity (irrespective of performance) in our prior paper (Turner and Levine, 2008). With respect to the current paper, the seed-behavior PLS identified connectivity patterns that were both related to and not related to performance. As seen in Figure 3 of our original work (Turner et al., 2011), whole-brain functional connectivity with the left BA 44 seed was not correlated with behavior for all conditions except for the Alphabetize 3 condition in patients with TBI. Regions functionally connected to the left BA 46 ROI were also not correlated with performance for the Alphabetize 5, Maintain 3, and Maintain 5 conditions in the TBI group. This is a key point of our paper: following brain injury, some functional connections within PFC are related to performance, and some are not. Moreover, PLS is a data-driven approach. It is entirely possible, if there was brain reorganization, 
for this analysis approach to yield a latent variable delineating areas of connectivity that were both functionally connected and behaviorally relevant in TBI and not in controls. This pattern did not emerge in the data-driven analysis, but there was nothing in this approach to prevent such a pattern from emerging if it was present.

(4) “The results from this approach may be misleading given that variance in accuracy data is artificially restricted in fMRI studies; investigators must guarantee high accuracy rates to ensure task compliance."

All groups were off ceiling for all conditions, except for the controls in the Maintain 3 condition. As discussed in Note 1 (p. 5) even here, the brain-behavior correlations were significant. While interpretation of the Maintain 3 condition should take this restriction of variance into consideration, this does not affect the interpretation of results from other conditions, which were key to overall interpretation of the brainbehavior correlations.

(5) The authors argue that recruitment of resources after injury can be compensatory, but only under some conditions. For example, they conclude that neural recruitment at lower task loads may be compensatory, but that increased prefrontal involvement at higher task loads in TBI is attributable to "poor regulation of functional brain activity."

We actually hold that altered functional engagement is compensatory not just at the earlier levels of task difficulty, but also at the higher levels, as illustrated in Figure 3 (Turner et al., 2011), where positive correlations with seed-connectivity can be seen in TBI patients across all levels of task difficulty. We have previously framed augmented activation as reflecting a generalized reduction in efficiency of brain function at all levels. That is, the system is not well regulated if it is engaged at lower levels of task demand (Turner and Levine, 2008). While our statement of poor regulation in response to increasing task loads may have been read as reflecting a viewpoint that the poor regulation applies only to higher task demands, this was not our intention.

(6) "It is equally dubious that functional engagement of prefrontal areas differs at each task load yet activation at all loads remains correlated with the same performance variable."
The fundamental principle behind this assertion relates to degeneracy (Mesulam, 1998; Price and Friston, 2002), which holds that there is more than one way for the brain to accomplish the same behavior, and also that a single brain systems can be engaged in multiple tasks. There are redundant systems for even basic functions, such as vision or perception, that can be differentially engaged to accomplish the same task. The application of this principle broadens when considering the same behavior across conditions as in our task, where we highlight differential brain network connectivity supporting accuracy in conditions of high versus low executive demand.

\section{SUMMARY}

We are grateful for the opportunity to clarify the misconceptions contained in Hillary's commentary. We agree that there are multiple routes to understanding altered functional brain activity in TBI and other patient groups. There is no standard analytical framework that is broadly accepted. We applied one approach that has been successfully applied in other patient groups (Grady et al., 2003a,b) and in manipulations with healthy subjects (for review, see McIntosh et al., 2004; Krishnan et al., 2011). There is no approach that is without limitations. We are encouraged by the recent increase in research in this domain and welcome the alternative frameworks as applied by other researchers.

\section{REFERENCES}

Backman, L., and Dixon, R. A. (1992). Psychological compensation: a theoretical framework. Psychol. Bull. 112, 259-283.

Bookheimer, S. Y., Strojwas, M. H., Cohen, M. S., Saunders, A. M., Pericak-Vance, M. A., Mazziotta, J. C., and Small, G. W. (2000). Patterns of brain activation in people at risk for Alzheimer's disease. New Engl. J. Med. 343, 450-456.

Buckner, R. L., Corbetta, M., Schatz, J., Raichle, M. E., and Petersen, S. E. (1996). Preserved speech abilities and compensation following prefrontal damage. Proc. Natl. Acad. Sci. U.S.A. 93, 1249-1253.

Cabeza, R., Grady, C. L., Nyberg, L., McIntosh, A. R., Tulving, E., Kapur, S., Jennings, J. M., Houle, S., and Craik, F. I. M. (1997). Age-related differences in neural activity during memory encoding and retrieval: a positron emission tomography study. J. Neurosci. 17, 391-400.

Engelien, A., Slibersweig, D., Stern, E., Huber, W., Döring, W., Frith, C., and Frackowiak, R. S. J. (1995). The functional anatomy of recovery from auditory agnosia: a PET study of sound categorization in a neurological patient and controls. Brain 118, 1395-1409.
Grady, C. L., McIntosh, A. R., Beig, S., Keightley, M. L., Burian, H., and Black, S. E. (2003a). Evidence from functional neuroimaging of a compensatory prefrontal network in Alzheimer's disease. J. Neurosci. 23, 986-993.

Grady, C. L., McIntosh, A. R., and Craik, F. I. (2003b). Age-related differences in the functional connectivity of the hippocampus during memory encoding. Hippocampus 13, 572-586.

Hillary, F. G. (2011). Determining the nature of prefrontal cortex recruitment after traumatic brain injury: a response to Turner. Front. Sys. Neurosci. 5:24. doi: 10.3389/fnsys.2011.00024

Krishnan, A., Williams, L. J., McIntosh, A. R., and Abdi, H. (2011). Partial least squares (PLS) methods for neuroimaging: a tutorial and review. Neuroimage 56, 455-475.

Levine, B., Black, S. E., Cabeza, R., Sinden, M., Mcintosh, A. R., Toth, J. P., Tulving, E., and Stuss, D. T. (1998). Episodic memory and the self in a case of isolated retrograde amnesia. Brain 121, 1951-1973.

McIntosh, A. R., Chau, W. K., and Protzner, A. B. (2004). Spatiotemporal analysis of event-related fMRI data using partial least squares. Neuroimage 23, 764-775.

Mesulam, M. M. (1998). From sensation to cognition. Brain 121, 1013-1052.

Price, C. J., and Friston, K. J. (2002). Degeneracy and cognitive anatomy. Trends Cogn. Sci. (Regul. Ed.) 6, 416-421.

Reuter-Lorenz, P. A., Jonides, J., Smith, E. E., Hartley, A., Miller, A., Marshuetz, C., and Koeppe, R. A. (2000). Age differences in the frontal lateralization of verbal and spatial working memory revealed by PET. J. Cogn. Neurosci. 12, 174-187.

Robertson, I. H., and Murre, J. M. (1999). Rehabilitation of brain damage: brain plasticity and principles of guided recovery. Psychol. Bull. 125, 544-575.

Rypma, B., and D'Esposito, M. (2000). Isolating the neural mechanisms of age-related changes in human working memory. Nat. Neurosci. 3, 509-515.

Turner, G. R., and Levine, B. (2008). Augmented neural activity during executive control processing following diffuse axonal injury. Neurology 71, 812-818.

Turner, G. R., McIntosh, A. R., and Levine, B. (2011). Prefrontal compensatory engagement in TBI is due to altered functional engagement of existing networks and not functional reorganization. Front. Syst. Neurosci. 5:9. doi: 10.3389/fnsys.2011.00009

Weiller, C., Chollet, F., Friston, K. J., Wise, R. J. S., and Frackowiak, R. S. J. (1992). Functional reorganization of the brain in recovery from striatocapsular infarction in man. Ann. Neurol. 31, 463-472.

Wood, F., McHenry, L., Roman-Campos, G., and Poser, C. M. (1980). Regional cerebral blood flow response in a patient with remitted global amnesia. Brain Lang. 9, 123-128.

Received: 22 January 2012; accepted: 09 February 2012; published online: 07 March 2012.

Citation: Turner GR, McIntosh AR and Levine B (2012) Dissecting altered functional engagement in TBI and other patient groups through connectivity analysis: one goal, many paths (a response to Hillary). Front. Syst. Neurosci. 6:10. doi: 10.3389/fnsys.2012.00010

Copyright (c) 2012 Turner, McIntosh and Levine. This is an open-access article distributed under the terms of the Creative Commons Attribution Non Commercial License, which permits non-commercial use, distribution, and reproduction in other forums, provided the original authors and source are credited. 